\section{Facultades de Odontología inclusivas. Estado del arte en Latinoamérica}

\author{
Inclusive Dentistry faculties. State of the \\ art in Latin America
}

\section{Resumen}

Las personas con discapacidad son una población vulnerable y excluida de sus derechos como el ingreso, la permanencia y el egreso de la educación superior, especialmente de las facultades de Odontología en Latinoamérica. El objetivo de esta revisión es hacer una revisión de la literatura acerca de la inclusión educativa en las facultades de Odontología en América Latina. Se realizó una revisión de la literatura nacional y latinoamericana alrededor de los conceptos discapacidad, educación inclusiva y pregrados de Odontología, con fuentes primarias, secundarias y obras de referencia; entre las cuales se consultaron, libros, tesis y base de datos Medline, SciELO, Pubmed, Lilacs, Dialnet y Redalyc. Se concluyó que las facultades de Odontología están en mora y en deuda con las personas con (dis)capacidad en Latinoamérica por las dinámicas de la educación superior que siguen perpetuando la exclusión o la discriminación de personas con discapacidad en los pregrados de Odontología.

Palabras clave: Atención dental para personas con discapacidades; Docentes de odontología; Educación en odontología; Estudiantes de odontología; Odontología (fuente: DeCS BIREME).

\begin{abstract}
People with disabilities are a vulnerable and excluded population from their rights such as admission, permanence and graduation from higher education, especially from in Latin American dental schools. The objective of this review is to review the literature on educational inclusion in Latin American dental schools. A review of the national and Latin American literature was carried out on the concepts of disability, inclusive education and undergraduate dentistry using primary and secondary sources; among which, books, thesis and Medline, SciELO, Pubmed, Lilacs, Dialnet and Redalyc databases were consulted. It was concluded that dental schools are in default and in debt to people with (dis) ability in Latin America due to the dynamics of higher education that continue to perpetuate the exclusion or discrimination of people with disabilities in undergraduate dentistry.
\end{abstract}

Keywords: Dental care for disabled; Dental faculty; Dental education; Dental students; Dentistry (source: MeSH NLM).

\section{Artículo de Revisión}

\author{
Ángela Liliana Grandas Ramírez ${ }^{1, a, b}$ \\ ${ }^{1}$ Universidad Nacional de Colombia, Facultad de Odonto- \\ logía, Bogotá, Colombia. \\ a Especialista en Estomatología Pediátrica. Magíster en \\ Discapacidad e Inclusión Social \\ ${ }^{b}$ Miembro Fundador de la Academia Interamericana de \\ Odontología para Pacientes Especiales (AIOPE) y de la \\ Asociación Colombiana de Odontología para Personas \\ con Diversidad Funcional (ACOPDIF).
}

\section{Correspondencia:}

Ángela Liliana Grandas Ramírez: algrandas@unal.edu.co Facultad de Odontología, Universidad Nacional de Colombia, Ciudad Universitaria. Carrera 30 con calle 45, Bogotá, D.C, Colombia, Código postal: 111321 ORCID: 0000-0002-5661-2853

\section{Editora:}

Rosse Mary Falcón-Antenucci

Universidad Inca Garcilaso de la Vega, Lima, Perú.

Conflicto de intereses: los autores declaran no tener conflictos de interés.

\section{Fuente de financiamiento: ninguna.}

Recibido: 19/11/20

Aceptado: 08/01/21

Publicado: 15/02/21 


\section{Introducción}

La (dis)capacidad se ha entendido desde varios aspectos de la evolución humana, como resultado del estudio de marcadores como las condiciones sociodemográficas, religiosas, científicas, técnicas, sociales, entre otras ${ }^{1}$. La convención la analiza como: "Las personas con (dis)capacidad incluyen a aquellas que tengan deficiencias físicas, mentales, intelectuales o sensoriales a largo plazo que, al interactuar con diversas barreras, puedan impedir su participación plena y efectiva en la sociedad, en igualdad de condiciones con las demás" ${ }^{2}$.

La educación inclusiva es acceder a este nivel de educación superior, es tener la oportunidad de desarrollar habilidades sociales, aumentar los conocimientos y cualificarse para ingresar al mundo laboral ${ }^{3}$ y está contemplado dentro de los derechos sociales que tiene cualquier persona como ciudadano ${ }^{4}$; rompiendo paradigmas, donde se valora la riqueza de la diversidad en medio de una educación accesible física y comunicativa ${ }^{5}$.

En la Facultad de Odontología de la Universidad Nacional de Colombia (FOUN), se piensa en las personas con (dis)capacidad desde el ámbito de la atención odontológica y desde el currículo, el cual se organiza no para asumir las diferencias sino para regularlas en función de formas de estratificación y jerarquización social y cultural ${ }^{5}$; desde los paradigmas del perfil de egresado de un talento humano en salud, los odontólogos no deben tener ningún tipo de (dis)capacidad.

Desde el punto de vista investigativo, son considerados como sujetos investigados y no como co-autores e innovadores en tecnología en y para la odontología y a nivel profesional no se las considera como colegas ejercedores de las ciencias odontológicas ${ }^{6}$. El derecho a la educación no se observa al interior de todas las instituciones de educación superior (IES), especialmente en las facultades de Odontología impidiendo su participación en la sociedad; no obstante no hay suficiente evidencia científica que visibilice la inclusión educativa a nivel de pregrado y menos a nivel de postgrado en Latinoamérica. El objetivo es hacer una revisión de la literatura acerca de la inclusión educativa en las facultades de Odontología en América Latina.

\section{Conceptualizaciones de la (dis)capacidad a través de la historia}

El concepto de la (dis)capacidad ha evolucionado; Palacios $^{7}$, devela los modelos de la prescindencia, el modelo rehabilitador y el modelo social y Peńas ${ }^{8}$ devela los modelos relacional y el modelo universalizante (Figura 1).

La conceptualización de la discapacidad en países del cono sur se ilustra específicamente en la tabla 19 .
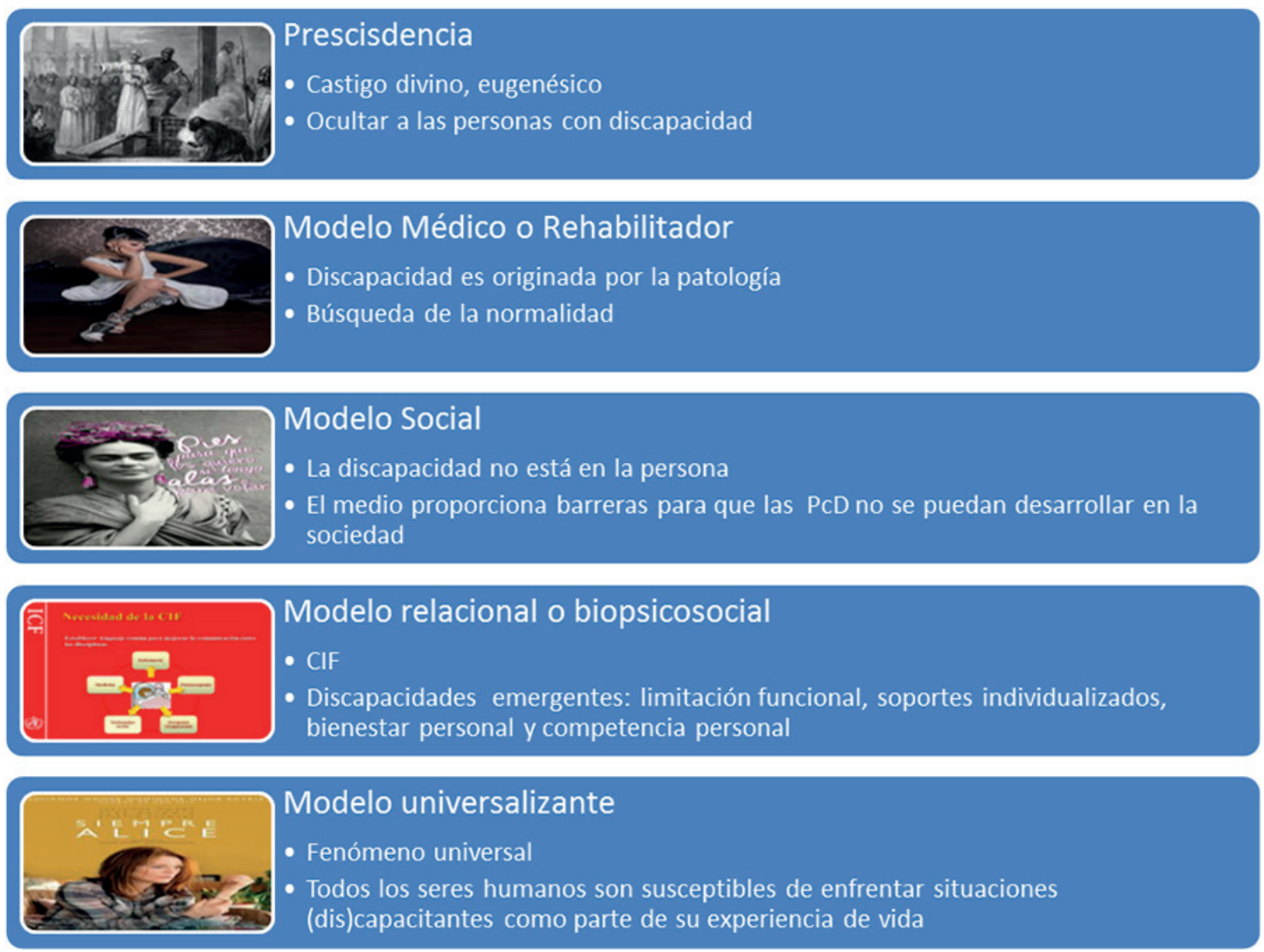

Figura 1. Conceptualizaciones de (dis)capacidad según Palacios y Peñas-Felizzola 7,8 
Tabla 1. Conceptualización en la educación superior a partir de Tejada9

\begin{tabular}{cllll}
\hline \multicolumn{1}{c}{ País } & \multicolumn{1}{c}{$\begin{array}{c}\text { Conceptualización de la } \\
\text { discapacidad }\end{array}$} & \multicolumn{1}{c}{ Noción de la inclusión } & Equidad & Diversidad \\
\hline Argentina & Enfoque interdisciplinario & Inclusión social y educativa & Igualdad de oportunidades & $\begin{array}{l}\text { Reconoce la diferencia y los } \\
\text { derechos }\end{array}$ \\
Chile & $\begin{array}{l}\text { Enfoque médico asociado } \\
\text { a necesidades especiales } \\
\text { educativas }\end{array}$ & $\begin{array}{l}\text { Inclusión educativa con el } \\
\text { fin de atenuar desigualda- } \\
\text { des sociales y económicas }\end{array}$ & $\begin{array}{l}\text { Equiparación de oportuni- } \\
\text { dades }\end{array}$ & $\begin{array}{l}\text { Interculturalidad referida a } \\
\text { as etnias }\end{array}$ \\
Uruguay & $\begin{array}{l}\text { Enfoque social como grupo } \\
\text { desfavorecido }\end{array}$ & $\begin{array}{l}\text { Política educativa que } \\
\text { responde a las demandas } \\
\text { sociales }\end{array}$ & Igualdad de oportunidades & $\begin{array}{l}\text { Variedad humana como } \\
\text { valor social }\end{array}$ \\
\hline
\end{tabular}

\section{Normatividad}

Las personas con (dis)capacidad están amparadas bajo legislaciones nacionales como internacionales, como fruto de la lucha de estas personas en su reconocimiento como sujetos de derecho: el movimiento de lucha (España) durante el foro de vida independiente, resaltó la noción de diversidad funcional; "con el fin de comprender que las personas funcionan de una manera diferente o diversa que la mayoría de la sociedad" ${ }^{10}$; es así como a nivel internacional surge la Convención de los derechos de las personas con discapacidad; en Colombia la Ley $1618^{2,11}$ (Tabla 2); en Argentina, se promulga en el 2006 la educación especial, como eje transversal al sistema educativo argentino, en Perú la Ley general de educación, Ley No. 28044: Acuerdo Nacional 20022021 acceso universal a una educación pública gratuita y de calidad ${ }^{12}$ y en Chile la Ley 20422 (10-FEB-2010), establece normas sobre igualdad de oportunidades e inclusión social de personas con discapacidad. Título IV párrafo $2^{\circ}$ de la educación y de la inclusión escolar.

\section{Educación inclusiva}

El pensamiento de una educación inclusiva es un proceso ineludible, ha sido adoptado por organismos como la Organización de las Naciones Unidas para la Educación, la Ciencia y la Cultura, con soporte jurídico, doctrinal y juridisprudencial ${ }^{10,13}$. Para logar este camino de la educación inclusiva debemos dejar de pensar en la educación superior como un proceso de solo transmisión de conocimientos y habilidades a las personas y replantear que las personas con discapacidad puedan empoderarse para significar sus propias decisiones.

La inclusión educativa, cruza el puente de considerar a la persona como sujeto, el cual debe integrarse y es el origen del problema; a pasar a un contexto en conjunto donde "la inclusión más como una actitud, una forma de sentir, una forma de valorar, más que de creencias" 13; para llegar como dice Marta Infante en Chile, a una educación accesible; sin embargo las personas no ingresan a la universidad por el resultado de la pobreza, la discapacidad y la marginalidad ${ }^{14}$.

Para las personas con discapacidad no logran el acceso, la permanencia y egreso de la Educación Superior (Informe sobre la Educación Superior en América Latina y el Caribe 2000-2005) '; esta afirmación se aprecia en la región de América Latina y el Caribe y más compleja debido a las desigualdades sociales de la región ${ }^{9}$. La Organización de las Naciones Unidas para la Educación, la Ciencia y la Cultura (UNESCO) creó el observatorio de educación inclusiva, pensando la educación como un bien público ${ }^{15}$. Pero al ser inclusiva, la educación no se plantea en términos de "necesidades, sino de oportunidades de cambio que giran en torno a unas características que tienen como base esencial los derechos humanos" ${ }^{16}$.

Las personas con discapacidad a través de la historia han vivido dos exclusiones: por un lado la segregación social, y por el otro, la homogeneización ${ }^{5}$. En la Universidad de Buenos Aires, el 17 de septiembre de 2003 el Consejo Superior sancionó la Resolución No 1.859/2003 basándose en: "Las personas con discapacidades y/o necesidades educativas especiales para ejercer su derecho a aprender en los ámbitos universitarios requieren apoyos que permitan eliminar o sobrellevar obstáculos para el desarrollo de la vida académica" ${ }^{17}$, por medio de la accesibilidad plena, física, comunicacional, cultural y pedagógica; un ejemplo es el Consejo Directivo de la Facultad de Filosofía y letras que aprobó el Proyecto del Programa de discapacidad e inclusive ya tiene su revista de universidad y discapacidad.

En la Universidad Nacional de la Plata se ha trabajado en "la transformación y eliminación de barreras no solo arquitectónicas, sino comunicacionales, actitudinales y académicas. Hay un acompañamiento e intento de resolución de situaciones individuales (por ejemplo, conseguir un apoyo a un estudiante -ya sea con un intérprete de Lengua de Señas o de la digitalización de un texto-, ..." ${ }^{18}$.

Si la Universidad Nacional de la Plata en Argentina considera que falta mucho por lograr y no están siendo inclusivos para todos, ¿Cómo estarán las facultades de Odontología en Latinoamérica? (Tabla 3)

\section{Cifras}

Para resolver este interrogante es necesario contextualizarnos con las cifras acerca de educación y discapacidad (tabla 4) ${ }^{19}$. Según la Organización de las Naciones Unidas ${ }^{20}$ :

- Tienen menor educación.

- El 90\% no asiste a la escuela. 
Tabla 2. Normatividad internacional y colombiana ${ }^{2,11}$

Convención de los derechos de las personas con discapacidad

Reconociendo la importancia de la accesibilidad al entorno

físico, social, económico y cultural, a la salud y la educación y a

la información y las comunicaciones, para que las personas con discapacidad puedan gozar plenamente de todos los derechos humanos y las libertades fundamentales

Artículo 24 Educación. Los Estados Partes reconocen el derecho de las personas con discapacidad a la educación. Con miras a hacer efectivo este derecho sin discriminación.

Los Estados Partes asegurarán que las personas con discapacidad tengan acceso general a la educación superior, la formación profesional, la educación para adultos y el aprendizaje durante toda la vida sin discriminación y en igualdad de condiciones con las demás. A tal fin, los Estados Partes asegurarán que se realicen ajustes razonables para las personas con discapacidad.
Ley Estatutaria 1618 de 2013

El Ministerio de Educación Nacional deberá, en relación con la educación superior: a) Consolidar la política de educación inclusiva y equitativa conforme al artículo 24 de la Convención de Naciones Unidas sobre los Derechos de las Personas con Discapacidad, la ley general de educación y los lineamientos de educación para todos de la UNESCO;

Artículo 11. Derecho a la Educación. El Ministerio de Educación Nacional definirá la política y reglamentará el esquema de atención educativa a la población con necesidades educativas especiales, fomentando el acceso y la permanencia educativa con calidad, bajo un enfoque basado en la inclusión del servicio educativo

Garantizar el personal docente para la atención educativa a la población con discapacidad, en el marco de la inclusión, así como fomentar su formación capacitación permanente, de conformidad con lo establecido por la normatividad vigente;

Emprender o promover la investigación y el desarrollo, y promover la disponibilidad y el uso de nuevas tecnologías, incluidas las tecnologías de la información y las comunicaciones, ayudas para la movilidad, dispositivos técnicos y tecnologías de apoyo adecuadas para las personas con discapacidad

b) Diseñar incentivos para que las instituciones de Educación Superior destinen recursos humanos y recursos económicos al desarrollo de investigaciones, programas, y estrategias para desarrollar tecnologías inclusivas e implementar el diseño universal de manera gradual;

Asegurar en todos los niveles y modalidades del servicio público educativo, que todos los exámenes y pruebas desarrollados para evaluar y medir la calidad y, cobertura, entre otros, así como servicios públicos o elementos análogos sean plenamente accesible a las personas con discapacidad

Incentivar el diseño de programas de formación de docentes regulares, para la inclusión educativa de la diversidad, la flexibilización curricular y en especial, la enseñanza a todas las personas con discapacidad, que cumplan con estándares de calidad

Asegurar, dentro del ámbito de sus competencias, a las personas con discapacidad el acceso, en condiciones de equidad con las demás y sin discriminación, a una educación superior inclusiva y de calidad, incluyendo su admisión, permanencia y promoción en el sistema educativo, que facilite su vinculación productiva en todos los ámbitos de la sociedad; en todo caso las personas con discapacidad que ingresen a una universidad pública pagarán el valor de matrícula mínimo establecido por la institución de educación superior

Tabla 3. Red Interuniversitaria Latinoamericana y del Caribe sobre Discapacidad ${ }^{3,9}$

\begin{tabular}{|c|c|c|c|}
\hline $\begin{array}{c}\text { País/ } \\
\text { Universidad }\end{array}$ & $\begin{array}{l}\text { Nombre de la RED/Año de } \\
\text { constitución }\end{array}$ & Actores & Articulación con otras redes \\
\hline $\begin{array}{l}\text { Argentina Universidad } \\
\text { Nacional de la Plata }\end{array}$ & $\begin{array}{l}\text { Red Interuniversitaria La- } \\
\text { tinoamericana y del Caribe } \\
\text { sobre Discapacidad y Derechos } \\
\text { Humanos (2009) }\end{array}$ & $\begin{array}{l}\text { Comité coordinador: Comisión } \\
\text { Interuniversitaria de Discapaci- } \\
\text { dad y Derechos Humanos }\end{array}$ & $\begin{array}{l}\text { Comisión Interuniversitaria de Discapa- } \\
\text { cidad y Derechos Humanos (Argentina) } \\
\text { *Institución Toledo de Ensino (Brasil) } \\
\text { *Red Colombiana de Universidades por } \\
\text { la Discapacidad } \\
\text { (Colombia) *Universidad Pedagógica } \\
\text { Nacional y UNAM (México) *Univer- } \\
\text { sidad Tecnológica de Panamá *Red } \\
\text { Temática de Discapacidad (Uruguay) }\end{array}$ \\
\hline $\begin{array}{l}\text { Chile Universidad de } \\
\text { Santiago de Chile }\end{array}$ & $\begin{array}{l}\text { Red de Educación Superior } \\
\text { Inclusiva (2008) }\end{array}$ & $\begin{array}{l}\text { *Representantes de las universi- } \\
\text { dades participantes *Autorida- } \\
\text { des del Servicio Nacional de la } \\
\text { Discapacidad }\end{array}$ & $\begin{array}{l}\text { *Redes Regionales: } \\
\text { - Norte } \\
\text { - Centro } \\
\text { - Sur }\end{array}$ \\
\hline $\begin{array}{l}\text { Uruguay Universidad } \\
\text { de la República }\end{array}$ & $\begin{array}{l}\text { Red Temática de Discapacidad } \\
(2008)\end{array}$ & $\begin{array}{l}\text { Coordinadores del programa, } \\
\text { estudiantes y comunidad } \\
\text { universitaria }\end{array}$ & $\begin{array}{l}\text { *Red Interuniversitaria Latinoameri- } \\
\text { cana sobre Discapacidad y Derechos } \\
\text { Humanos (Argentina) }\end{array}$ \\
\hline $\begin{array}{l}\text { Brasil Universidad } \\
\text { Federal de Río Grande } \\
\text { Do Sul }\end{array}$ & & $\begin{array}{l}\text { Red de Asambleas Pedagógicas } \\
\text { que regulan la política de los } \\
\text { sordos y son conformadas por } \\
\text { los estudiantes, padres, profeso- } \\
\text { res, intérpretes }\end{array}$ & \\
\hline
\end{tabular}


Tabla 4. Proporción de personas de 15-29 años edad con una discapacidad en la educación 2018, según UNESCO 19

\begin{tabular}{|c|c|c|c|c|c|}
\hline \multirow[t]{2}{*}{ País } & \multirow[t]{2}{*}{ Año } & \multicolumn{2}{|c|}{$\begin{array}{l}\text { Proporción de personas de edad } \\
\text { 15-29 años con una discapacidad }\end{array}$} & \multicolumn{2}{|c|}{$\begin{array}{l}\text { Alguna vez asististe a la educación, } \\
\text { personas con una discapacidad }\end{array}$} \\
\hline & & $\mathrm{H}$ & $\mathrm{M}$ & $\mathrm{H}$ & M \\
\hline Brasil & 2010 & $3,2 \%$ & $2,9 \%$ & $87.7 \%$ & $90.8 \%$ \\
\hline Argentina & & s.d & s.d & s.d & s.d \\
\hline Perú & 2013 & $1,9 \%$ & $1,5 \%$ & s.d & s.d \\
\hline Guatemala & & s.d & s.d & s.d & s.d \\
\hline Chile & & s.d & s.d & s.d & s.d \\
\hline Colombia* & 2013 & $2,3 \%$ & $2,7 \%$ & $94,5 \%$ & $99,5 \%$ \\
\hline
\end{tabular}

*Colombia 2013: Población entre los 14 y los 28 años: 12,571,280 jóvenes

H:Hombres: 6,403,564 - M:Mujeres: 6,167,716 22

H: Hombres, M: Mujeres, s.d: Sin datos

- Los adultos alfabetizados son: hombres $3 \%$ y mujeres $1 \%$.

- Pocos estudiantes tienen educación superior.

- El ingreso a las IES está influenciado por el nivel socioeconómico.

- Según la Unesco, en 49 países, las personas con discapacidad pasaron menos años en la escuela que sus contrapartes sin discapacidad. En promedio, en los 22 países y territorios con datos, las personas sin discapacidad tienen 7,0 años de escolaridad y las personas con discapacidades 4,8 ańos ${ }^{15}$.

- En Ecuador de 1608334 de personas con discapacidad, el $54 \%$ de esa población no tiene ningún tipo de educación formal, el 18\% tiene nivel de educación primaria, el 19\% educación secundaria y tan solo el $8 \%$ presenta educación superior ${ }^{21}$.

- En Colombia el 2013: a población entre los 14 y los 28 años fueron 12571280 y hombres: 6403564 y mujeres: $6167716^{22}$.

\section{¿Qué ocultan las cifras...develan barreras?}

¿Qué ocultan las cifras?; la población con (dis)capacidad no es garante de sus derechos con respecto a la educación; los Estados y las Instituciones de Educación Superior (IES) es probable que no proclaman los acuerdos como las Normas Uniformes sobre la Igualdad de Oportunidades para las Personas con Discapacidad, la Declaración de Derechos Humanos o la Declaración Mundial sobre Educación para Todos y la Declaración de Salamanca: "Los programas de estudios de los estudiantes con necesidades educativas especiales en clases superiores deberán incluir programas de transición específicos, apoyo para el ingreso en la enseñanza superior cuando sea posible, y la subsiguiente capacitación profesional para prepararlos a funcionar como miembros independientes y activos de sus comunidades al salir de la escuela" ${ }^{23}$.
El Informe mundial de discapacidad ${ }^{24}$, revela la falta de recurso humano y como la formación docente puede mejorar su confianza y sus aptitudes para educar a nińos con discapacidad, la falta de apoyo económico para implementar políticas, protección social, sensibilización pública y la comprensión de la discapacidad. En ocasiones no se hacen cumplir las políticas o las normas son insuficientes, otros aspectos como las creencias y prejuicios constituyen obstáculos para la educación, por ejemplo, las actitudes de los docentes y administrativos ${ }^{10,24}$, actitudes sociales desfavorables ${ }^{24-26}$ y factores externos como la inaccesibilidad física ${ }^{9,10,24}$, las regulaciones, sistemas y estrategias pedagógicas dominantes; y no se valoran las capacidades de las personas con discapacidad ${ }^{10}$, la existencia de discriminación que es el no reconocimiento de la identidad del otro ${ }^{27}$ y barreras de acceso a la educación formal donde las condiciones de acceso y permanencia en la educación son insuficientes ${ }^{26,28}$.

Otro factor que se considera barrera es la actitud de los estudiantes, en Chile se muestra como estudiantes cuando opinan sobre inclusión de personas con discapacidad, refieren en un bajo porcentaje, que prefieren no tener compañeros con discapacidad en clases ${ }^{26,29}$.

Otra barrera es el desconocimiento de las leyes por parte de los actores y de los beneficiarios; el deficiente ejercicio de la ciudadanía por parte de las propias personas con discapacidad ${ }^{9}$.

Las barreras propias de la educación superior tales como "los sistemas de acceso y de selección, la creciente privatización, la concentración de la oferta, la rigidez de los planes de estudio, de la evaluación, y las formas de enseñanza, y la falta de sistemas de apoyo para estudiantes y docentes" 30 .

\section{Más allá de la accesibilidad}

Dentro de los procesos enseńanza-aprendizaje se pueden utilizar los sistemas de ambientes virtuales de aprendizaje (AVA), modelos de enseñanza (e-learning, m-learning, b-learning $\mathrm{y}$, recientemente, u-learning ${ }^{31}, \mathrm{y}$ tener en cuenta la guía de accesibilidad pensada desde la UNESCO ${ }^{32}$. 


\section{Vislumbrando nuevos roles del profesorado}

Los maestros son el factor más influyente para determinar la equidad, acceso y calidad en educación, y representan la clave del desarrollo sostenible y del desarrollo del planeta ${ }^{33}$. El personal académico, como lo denota Martín-Padilla et al. ${ }^{10}$, no debe asumir toda la carga en capacitarse, pero sí debe sensibilizarse ante los conceptos de discapacidad ${ }^{34}$. La investigación sobre la formación de profesores para la educación inclusiva se puede fundamentar en valores que sustentan la competencia de los ellos en el desarrollo y mantenimiento de la práctica inclusiva: valorar la diversidad de los alumnos, apoyar a los estudiantes, trabajo en equipo y educación continuada ${ }^{35}$ (Tabla 5) ${ }^{36}$.

Los docentes requieren capacitación y apoyo de otros profesionales como los psicólogos para aprender a atender determinadas circunstancias ${ }^{36}$.

Otra consideración a tener en cuenta, es el rompimiento del paradigma del profesor, de que hay que categorizar a la persona basada en el diagnóstico y tratamiento médico - modelo rehabilitador- como primer paso para proporcionar bienes educativos adecuados a estudiantes con discapacidad; y no desde su funcionamiento atendiendo a la Clasificación Internacional del Funcionamiento, de la Discapacidad y de la Salud (CIF), sino desde su limitación ${ }^{1,14}$. Asimismo Underwood ${ }^{17}$ habla de respetar las diferencias, no de tolerarlas.

Un desafío que se vive en Chile y otros países latinoamericanos es gran parte del currículum de educación superior incluye herramientas específicas a nivel de pedagogías y enseńanzas que se centran en el aprendizaje de un estudiante promedio; sin olvidar que "las competencias que definen a un profesor o profesora, delimitadas en su currículum de pregrado, no necesariamente son aquellas que el mismo estudiante en formación ha elaborado a través de sus experiencias educativas" ${ }^{14}$. A pesar de las modificaciones que se han realizado en la educación superior en Chile, no han generado cambios profundos en los procesos de inclusión educativa ${ }^{14}$.

Se podría apoyar el pensamiento de Bustamante expuesto por Díaz 5, "lo mínimo esperable y demandable de un docente es una actitud, un posicionamiento y/o una idiosincrasia que implica asumir en su trabajo: el reconocimiento de la diversidad de códigos culturales”.

La evaluación se podría replantear desde una "evaluación cualitativa por procesos implica el reconocimiento de diversos momentos por los cuales transita el sujeto en formación; estos momentos se podrían distinguir en función de niveles de complejidad cualitativamente diferenciables" 5 , lo que conlleva a unas formas de sistematización, seguimiento, socialización y retroalimentación de las producciones y procesos; y desde esta perspectiva, "se propone una comprensión de la evaluación desde una visión amplia, compleja, históricamente situada, contextualizada, en la que se reconocen sus múltiples aristas, su polifonía y la diversidad de sentidos que la configuran como espacio aún por construir" "; no vistas en las facultades de odontología, las cuales generaran múltiples tensiones.

\section{¿Cómo transitan las facultades de Odontología den- tro de la inclusión educativa?}

En el libro Guía para asegurar la inclusión y la equidad en la educación de la UNESCO del $2017{ }^{35}$, no se visibiliza la inclusión educativa en las facultades de Odontología.

Según Molina en el $2005^{3}$, en su trabajo de investigación sobre integración educativa en la Universidad Nacional de Colombia; referente a Odontología vislumbró en ese año: los vicedecanos tienen conocimiento de la existencia de estudiantes con discapacidad en sus facultades (Odontología, Enfermería y Medicina) refieren que ofrecen apoyo individualizado al estudiante, en términos de acompañamiento académico y consecución de recursos económicos. En Odontología los registros no coinciden con la realidad, además refieren que en sus facultades no conocen la existencia de personal con formación en materia de integración educativa para personas con discapacidad. Evidencian que existe mínima accesibilidad en el entorno físico del campus universitario y especialmente al interior de las facultades.

Una encuesta semiestructurada a miembros de Academia Interamericana de Odontología Pacientes Especiales (AIOPE) y Asociación Colombiana de Odontología para Personas con Diversidad Funcional (ACOPDIF),

Tabla 5. Nuevas tendencias en desarrollo profesional para docentes en América Latina, según Valliant ${ }^{36}$

\begin{tabular}{lll}
\hline \multicolumn{1}{c}{ País } & \multicolumn{1}{c}{ Programa } \\
\hline Brasil & Accelerated learning & Innovación \\
Colombia & Escuela Nueva & Grupos de discusión de casos de estudio \\
Costa Rica & Educational technology & Tecnología en la formación docente \\
Perú & Teacher training (PLANCAD) & Experiencias compartidas \\
Venezuela & Fe y Alegría & Enfoques basados en el aula \\
\hline
\end{tabular}

La inclusión con calidad, interculturalidad y el fortalecimiento de las Cátedras UNESCO de Inclusión en Educación universitaria superior para entrenar aprendices sobre liderazgo y derechos, y publicar y diseminar informes sobre acceso e inclusión.

El éxito del programa de becas basado en Chile y con el apoyo de la UNESCO Santiago llevó a su replicación en otros países de la región.

La UNESCO apoya Secretaría de Educación del Distrito Bogotá, D. C., Colombia 
en enero del 2018 nos muestra en la figura 2, la poca población con discapacidad que ingresa y egresan en las facultades de odontología en América Latina; siendo las facultades de Odontología de Brasil: Uniagrario de Janeiro, UFAIPE y Univeridade do estado do Amazonas y de Argentina: UnCuyo en Mendoza (Argentina) las más inclusivas, realizando ajustes razonables como adaptaciones al examen de ingreso, adaptaciones a los exámenes escrito y orales, acondicionamiento físico de las unidades odontológicas y un acompańamiento integral lo que ha permitido el egreso de personas con discapacidad de las facultades de odontología, dos en UnCuyo Mendoza y 40 con necesidades especiales (enfermedades crónicas y discapacidad) en Brasil. En la Universidad Nacional de Colombia han estudiado dos personas con discapacidad, se desconoce el número de egresados.

Se ha develado que estudiantes de Odontología presentan discapacidades en hombros, brazo y manos ${ }^{37}$ y problemas de aprendizaje como dislexia, disgrafía y discalculia ${ }^{38}$.

\section{Recomendaciones}

Sensibilizar a la comunidad universitaria en sus tres estamentos, personal docente, personal administrativo y estudiantado en la discapacidad desde la perspectiva de interseccionalidad.
Desde bienestar universitario, mantener el apoyo psicosocial a los estudiantes con (dis)capacidad. Crear una política de educación superior inclusiva y de bienestar al interior de las facultades de odontología, con la formulación de iniciativas, decisiones y acciones en discapacidad; basándose en la autonomía que presentan las IES; para ser tenido en cuenta en el plan de desarrollo de las facultades de odontología. Con soporte de la política institucional de cada Universidad; apoyado de un voluntariado social conformado por los propios estudiantes ${ }^{3}$. Incorporar la temática de discapacidad en la malla curricular ${ }^{6}$; lo cual contribuiría a la formación de profesionales íntegros y facilitadores de inclusión en el futuro como lo afirma la UNESCO. Reconocer la "diversidad como un valor y no como un defecto implica romper con la clasificación y la norma, supone plantearnos una necesaria profesionalización del docente para la comprensión de la diversidad y requiere pensar en un currículo que, ahondando en las diferencias del alumnado, erradique las desigualdades a la vez que haga avanzar la justicia escolar ofreciendo prácticas educativas simultáneas y diversas" 39.

Durante la construcción de la reforma académica en las facultades de odontología se pueden considerar las preguntas orientadoras de la UNESCO, tales como:

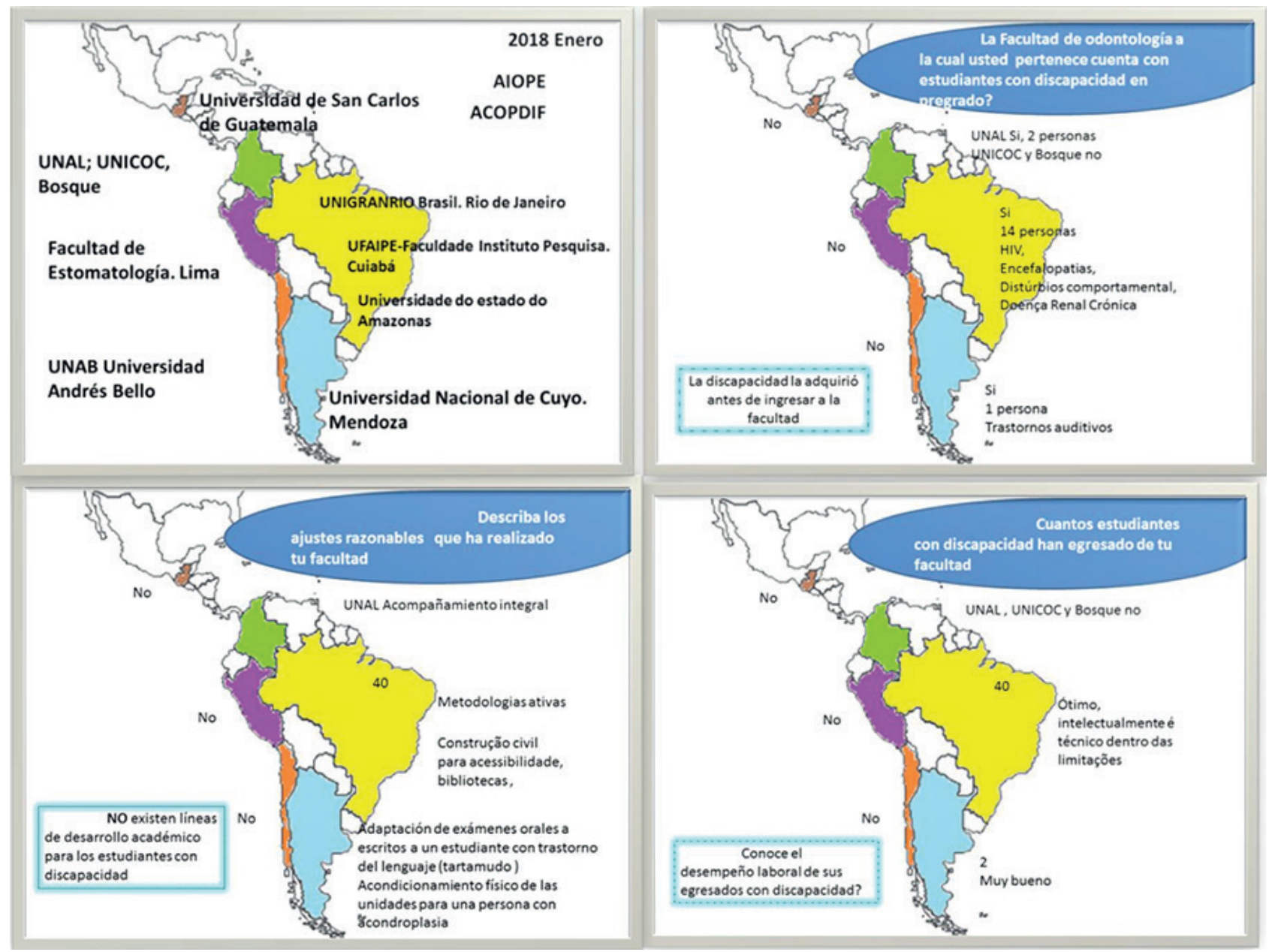

Figura 2. Educación inclusiva en facultades de Odontología en América Latina 
- ¿En qué medida el currículo nacional se basa en los principios de inclusión y equidad?

- ¿En qué medida el currículo nacional tiene la solidez y la flexibilidad para adaptarse a todos los estudiantes?

- ¿En qué medida se utilizan los sistemas de evaluación para celebrar los diferentes niveles de logro y apoyar el desarrollo de todos los estudiantes?

Las facultades de Odontología como misión deberán apropiarse de la diversidad, diferencia y pluralidad, de la formación permanente en múltiples contextos, sistemas educativos flexibles, descentralización, institución educativa abierta e integrada 5 .

En Chile, los mismos estudiantes proponen que, se crea una comisión universitaria sobre discapacidad y la necesidad que existan canales de comunicación más efectivos entre compañeros y compañeras como apoyo y ayuda a estudiantes con discapacidad ${ }^{14}$.

Acoger la disposición del "Primer seminario regional sobre la integración de las personas con discapacidad en la educación superior en América Latina, el cual recomendó que "la accesibilidad para personas con discapacidad sea requisito para acreditación universitaria. Decisión centrada especialmente en el diseño de planes estratégicos de acción que lleven a la práctica la valoración de la diferencia y la diversidad, así como el respeto de la dignidad de las personas con discapacidad como principios inseparables de la labor educativa" ${ }^{30}$.

Argentina propone que se debería lograr la construcción de personas con discapacidad creativos, reflexivos, dinámicos, propositivos, en definitiva, de sujetos políticos capaces de jugarse por sus perspectivas ocupacionales, capaces de exigir y de proponer horizontes de acción ${ }^{18}$.

La Facultad de Odontología de la Universidad Nacional se sugiere adoptar las acciones del Acuerdo 036 el 2012: preparación pedagógica, adquisición y uso de tecnologías de la información y comunicación, fortalecimiento de la corresponsabilidad institucional, diseño, adaptación e implementación de recursos para la movilidad, egreso y preparación para el cambio, accesibilidad física y producción académica en torno a la discapacidad ${ }^{40}$.

\section{Conclusión}

Las facultades de Odontología están en mora y en deuda con las personas con (dis)capacidad en Latinoamérica por las dinámicas de la educación superior que siguen perpetuando la exclusión o la discriminación de personas con discapacidad en los pregrados de Odontología.

\section{Referencias bibliográficas}

1. Bernal A, Moreno M. Educación inclusiva CIF-NJ y jóvenes con discapacidad intelectual [Internet]. Bogotá: Universidad Nacional de Colombia: Facultad de Medicina. Grupo de investigación Discapacidad, Políticas y Justicia Social; 2013. 100 p. Disponible en: https: https:// www.researchgate.net/publication/337498686_EDUCACION_INCLUSIVA_CIF_Y_JOVENES_CON_ DISCAPACIDAD_INTELECTUAL
2. Organización Naciones Unidas. Convención sobre los derechos de las personas con discapacidad [Internet]. 2006. Disponible en: https://www.un.org/development/desa/disabilities-es/convencion-sobre-los-derechos-de-las-personas-con-discapacidad-2.html

3. Molina R. Integración educativa de los estudiantes con discapacidad en la Universidad Nacional de Colombia Realidades y Retos. [Bogotá: Maestría Discapacidad e Inclusión Social]: Universidad Nacional de Colombia; 2005.

4. Fernández A. La ciudadanía para las personas con discapacidad. En: Hacia una universidad accesible Construcciones colectivas por la discapacidad [Internet]. Argentina: Universidad Nacional de La Plata (Edulp); 2011. p. 145-64. Disponible en: http://sedici.unlp.edu.ar/ bitstream/handle/10915/26982/Documento_completo. pdf?sequence $=1$

5. Díaz O. Hacia una concepción de la atención educativa de personas con discapacidad. Bogotá, Colombia: Ministerio de Educación Nacional; 2003 p. 113.

6. Grandas A. La discapacidad en perspectiva interseccional. Una ruta para transversalizar la inclusión epistémica en pregrados de Odontología [Internet] [Tesis Meritoria]. [Bogotá: Maestría Discapacidad e Inclusión Social]: Universidad Nacional de Colombia; 2015. Disponible en: bdigital.unal.edu.co/63386/1/52589724.2015.pdf

7. Palacios A. El modelo social de discapacidad: orígenes, caracterización y plasmación en la Convención Internacional sobre los Derechos de las Personas con Discapacidad [Internet]. 1ra Edición. Colección Cermies. Vol. 36. Madrid: Grupo editorial CINCA; 2008. Disponible en: https://www.google.com.co/search?$\mathrm{q}=$ agustina + palacios + discapacidad\&oq=Agustina+pala cios + \&aqs $=$ chrome $2.69 \mathrm{i} 57 \mathrm{j} 015.9790 \mathrm{j} 0 \mathrm{j} 4 \&$ sourceid $=-$ chrome\&ie $=$ UTF- 8

8. Peñas-Felizzola OL. Referentes conceptuales para la comprensión de la discapacidad. Rev Fac Med [Internet]. 2013;61(2):205-12. Disponible en: https://revistas.unal. edu.co/index.php/revfacmed/article/view/39696

9. Tejeda P. Aproximación a las políticas de educación superior para estudiantes con discapacidad en los países del cono sur: Argentina, Chile y Uruguay. [Bogotá: Maestría Discapacidad e Inclusión Social]: Universidad Nacional de Colombia; 2012.

10. Martín-Padilla E, Sarmiento PJ, Coy LY. Educación inclusiva y diversidad funcional en la Universidad. Rev Fac Med [Internet]. 2013;61(2):195-204. Disponible en: https://revistas.unal.edu.co/index.php/revfacmed/article/ view/39695

11. Congreso de Colombia. Ley Estatutaria 1618 de 2013 [Internet]. 2013. Disponible en: http://wsp.presidencia. gov.co/Normativa/Leyes/Documents/2013/LEY\%20 1618\%20DEL\%2027\%20DE\%20FEBRERO\%20 DE\%202013.pdf

12. Ley general de educación, Ley No. 28044 [Internet]. Disponible en: https://www.mimp.gob.pe/files/direcciones/dgfc/diff/normat_nacional_apafas/1_Ley_28044. pdf

13. García S, Fernández A. La inclusión para las personas con discapacidad: entre la igualdad y la diferencia. Rev Cienc Salud [Internet]. 2005;3(2):235-46. Disponible 
en: http://www.scielo.org.co/scielo.php?script=sci_abstract\&pid $=$ S1692-72732005000200013\&lng=en\&nr$\mathrm{m}=\mathrm{iso} \& \mathrm{t} \operatorname{lng}=\mathrm{es}$

14. Infante M. Desafíos a la formación docente: inclusión educativa. Estudios Pedagógicos [Internet]. 2010;XXXVI(1):287-97. Disponible en: https://scielo.conicyt.cl/ scielo.php?script=sci_arttext $\&$ pid $=$ S0718...

15. Organización Naciones Unidas. América Latina y el Caribe. Revisión general 2015 de la educación para todos. [Internet]. 2015 p. 375. Disponible en: http://unesdoc. unesco.org/images/0023/002327/232701s.pdf

16. Ministerio de Educación Nacional, Fundación Saldarriaga Concha. Índice de inclusión para educación superior (INES) [Internet]. Colombia; 2017.158 p. Disponible en: https://www.mineducacion.gov.co/1759/ articles-357277_recurso_1.pdf

17. Underwood S. UBA: Programa Discapacidad y Universidad. Revista Espacios. 2012;49:12-30.

18. Katz S, Danel P. Hacia una universidad accesible. Construcciones colectivas por la discapacidad. Argentina: Universidad Nacional de La Plata (Edulp); 2011. 201 p.

19. UNESCO Institute for Statistics. Education and Disability: Analysis of Data from 49 Countries [Internet]. 2018. Disponible en: uis.unesco.org/.../education-and-disability-analysis-data-49-count

20. Organización Naciones Unidas. Algunos datos sobre las personas con discapacidad Disabilities ES [Internet]. 2020. Disponible en: https://www.un.org/development/desa/disabilities-es/algunos-datos-sobre-las-personas-con-discapacidad.html

21. Miranda W. Políticas estatales y los paradigmas de la discapacidad: Tres experiencias de producción cultural. [Ecuador: Programa de Maestría en Estudios de la Cultura]: Universidad Andina Simón Bolívar Sede Ecuador; 2013.

22. DANE. Proyecciones de población jóvenes 14-28 años [Internet]. 2018. Disponible en: http://obs.colombiajoven.gov.co/Observatorio/Observatorio.aspx?rpt=Buyel7PqAptVw9lwUXO_Fw==

23. Organización Naciones Unidas. Declaración de Salamanca y marco de acción para las necesidades educativas especiales [Internet]. Salamanca, España: Organización de las Naciones Unidas para la Educación, la Ciencia y la Cultura y Ministerio de Educación y Ciencia, España; 1994 p. 49. Disponible en: http://www.unesco.org/education/pdf/SALAMA_S.PDF

24. Organización Naciones Unidas. Informe Mundial de la Discapacidad [Internet]. 2011 p. 27. Disponible en: http://www.who.int/disabilities/world_report/2011/ summary_es.pdf

25. Sen A. Discapacidad y justicia [Internet]. Segunda conferencia internacional sobre discapacidad y desarrollo inclusivo; 2004; Banco Mundial. Disponible en: www. ibrarian.net/navon/paper/DISCAPACIDAD_Y_JUSTICIA.pdf?paperid

26. Solís P, CEPAL. Discriminación estructural y desigualdad social. Con casos ilustrativos para jóvenes indígenas, mujeres y personas con discapacidad. Primera. Vol. Consejo Nacional para Prevenir la Discriminación. México: Fundamentos; 2017. 134 p.
27. Alzate JIC. Historicidad de procesos de exclusión y discriminación para grupos minoritarios en educación superior. Rev Senderos Pedagógicos ISSN 2145-8243 E-ISSN 2590-8456 [Internet]. 2017;8(8):141-53. Disponible en: https://ojs.tdea.edu.co/index.php/senderos/ article/view/534

28. Consejo Nacional de Política Económica y Social. Departamento Nacional de Planeación. Política pública nacional de discapacidad e inclusión social. Conpes Social 166 [Internet]. 2013. Disponible en: https://www. minsalud.gov.co/sites/rid/Lists/BibliotecaDigital/RIDE/ INEC/IGUB/CONPES166.pdf

29. Ocampo A. Inclusión de estudiantes en situación de discapacidad a la educación superior. Desafíos y oportunidades. Revista Latinoamericana de Educación Inclusiva [Internet]. 2013;6(2):227-39. Disponible en: http://riberdis.cedd.net/handle/11181/5553

30. Pérez L, Fernández A, Katz S. Discapacidad en Latinoamérica: Voces y experiencias universitarias [Internet]. Argentina: Editorial de la Universidad Nacional de La Plata (Edulp); 2013. 301 p. Disponible en: http://repositoriocdpd.net:8080/bitstream/handle/123456789/619/L_PerezL_DiscapacidadLatinoamericaUniversitarias_2013. pdf?sequence $=1$

31. Martínez M del C. Experiencias de inclusión educativa en Colombia: hacia el conocimiento útil. RUSC [Internet]. 2011;8(1):43-54. Disponible en: rusc.uoc.edu/ rusc/es/index.php/rusc/article/view/v8n1-martinez.html

32. UNESCO, IESALC. Propuesta de Guía de accesibilidad. Evaluación y control permanente de la: accesibilidad en la calidad de los servicios, accesibilidad en la comunicación e información, accesibilidad física de tránsito y permanencia y accesibilidad del uso de productos. 2011. $173 \mathrm{p}$.

33. UNESCO. Annual Report 2016. Regional Bureau for Education in Latin America and the Caribbean. Education 2030 [Internet]. 2017. Disponible en: http://www. unesco.org/new/es/media-services/single-view-tv-release/news/annual_report_2016_regional_bureau_for_ education_in_latin_al

34. Grandas A, Munévar DI. Inclusión epistémica de la discapacidad en el campo de la salud oral. Rev Colomb Salud Libre [Internet]. 2017;11(1):16-25. Disponible en: http://revistasojs.unilibrecali.edu.co/index.php/rcslibre/ article/view/247

35. Organización Naciones Unidas. Guía para asegurar la inclusión y la equidad en la educación [Internet]. París, Francia; 2017. 46 p. Disponible en: http://down21-chile.cl/cont/cont/2017/336_2_guia_para_asegurar_la_inclusion_y_la_equidad_en_la_educacion.pdf

36. Vaillant D. Preparing teachers for inclusive ducation in Latin America. Prospects Q Rev Comp Educ. 2011;41(3):385-98.

37. Wajngarten D, Campos JADB, Botta AC, Nordi Sasso Garcia PP. Validity and reliability of the disabilities of arm, shoulder, and hand scale in dental students: A transnational study. Arch Environ Occup Health. 2018;73(4):258-66.

38. Cruikshank D, Howell TH, Brinckerhoff LC, Badovinac $\mathrm{R}$, Karimbux NY. Learning disabilities in dental educa- 
tion: trends, management, and concerns in U.S. dental schools. J Dent Educ. 2002;66(10):1178-84.

39. Ministerio de Educación, Cultura y Deporte. Educación Inclusiva. Iguales En la diversidad. Clarificar nuestros valores. Igualdad en la Diversidad. 2012;14.
40. Consejo Superior Universitario. Acuerdo 036 del 2012. Por el cual se establece la política institucional para la inclusión educativa de las personas con discapacidad en la Universidad Nacional de Colombia. 2012 p. 2. 